\title{
Gastric Diverticulum in Computed Tomography
}

\author{
Feyza Sönmez Topçu ${ }^{1}$ (D), Umit Koç ${ }^{2}$ (iD) \\ ${ }^{1}$ Department of Radiology, ISU Bahcesehir Liv Hospital, Istanbul, Turkey \\ ${ }^{2}$ Department of General Surgery, ISU Bahcesehir Liv Hospital, Istanbul, Turkey
}

\begin{abstract}
Gastric diverticula are the outpouchings of the stomach wall. They are the least common diverticula of the gastrointestinal system. They are usually asymptomatic and are diagnosed accidentally by upper gastrointestinal contrast radiographic studies or upper gastrointestinal endoscopy. Identification is important as they can cause serious complications such as upper gastrointestinal bleeding or perforation. We herein present a case of asymptomatic congenital gastric diverticulum.
\end{abstract}

Turk J Int Med 2020;2(4):127-130

DOI: $\underline{10.46310 / \text { tjim.772179 }}$

Keywords: gastric diverticulum, stomach diverticulum, diverticula, endoscopy

\section{Introduction}

Gastric diverticula (GD) are very rare anomalies seen in upper gastrointestinal system (GIS) contrast radiographic studies (0.04\%), in upper GIS endoscopy examinations (0.01$0.11 \%$ ) and in autopsy series $(0.02 \%) .{ }^{1-3}$ They are similar structures with small intestine and colon diverticula and seen equally in men and women between the ages of 50-70. Although they are generally asymptomatic, sometimes cause symptoms such as epigastric pain, discomfort, nausea, vomiting, halitosis, bloating, early satiety, anorexia and dysphagia. ${ }^{3} \mathrm{GD}$ greater than $4 \mathrm{~cm}$ in diameter are more susceptible to complications and are less likely to respond favorably to medical management. ${ }^{3}$

Since the symptoms are uncertain and have no distinctive findings from other GIS diseases, the most important step to diagnose the disease is to keep this pathology in mind and in the list of possible diagnoses. Follow-up or palliative treatment options such as proton pump inhibitors and antacid treatments are preferred in asymptomatic patients, surgical treatment is required if serious complications such as ulceration, malignant transformation or perforation occur in the gastric diverticulum. 


\section{Case Report}

Our patient is a 30-year-old woman. When she applied to the Internal Diseases outpatient clinic with a complaint of dyspnea, thorax computerized tomography (CT) study was performed. CT scan showed an incidental stomach diverticulum with $3 \times 2 \mathrm{~cm}$ size in the gastric fundus posterior wall (Figure $1 a$ and $1 b$ ). Although it was very close to the left adrenal gland, the continuity of diverticulum with the stomach lumen, air density (Figure 2) in it and the fat line between the lateral adrenal crus could be seen (Figure 3). After CT study, upper GIS endoscopy was performed and wide-mouthed diverticulum was observed in the fundus (Figure 4).

Since our patient was asymptomatic and pathologies such as ectopic mucosa, ulcer or malignancy were not found in the gastric diverticulum, clinical monitoring without treatment advised. Informed consent was taken before all procedures.

\section{Discussion}

GD can be classified as congenital or true diverticulum and acquired or false diverticulum. True or congenital diverticula are mostly located on the posterior wall of the gastric fundus, contain all the layers of the gastric wall and are thought to be associated with the defect in the fusion of the dorsal and ventral mesentery in the embryonic period. ${ }^{5}$ Seventy-five percent of them are located in fundus, $2 \mathrm{~cm}$ below the gastroesophageal junction on the posterior wall, $3 \mathrm{~cm}$ from the small curvature, and

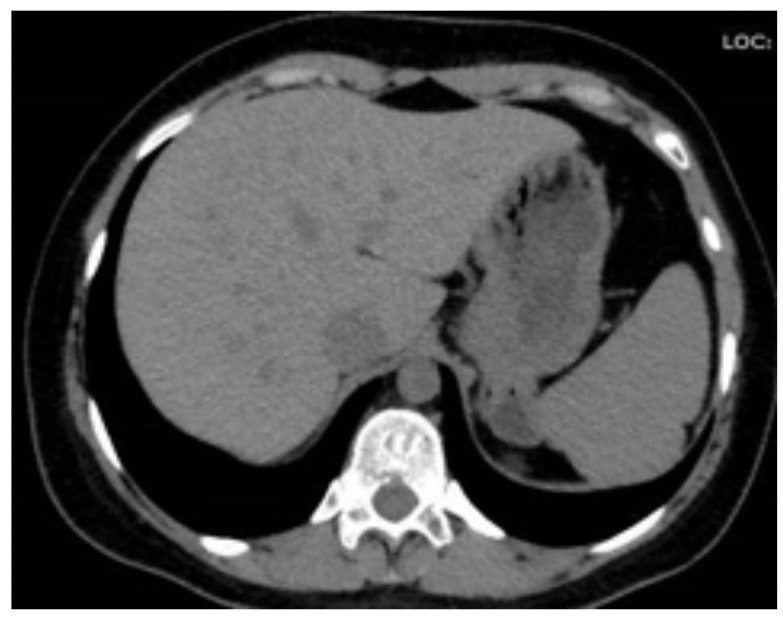

Figure 1a: CT-scan-axial sections, GD continuous with stomach lumen their diameter ranges from $3 \mathrm{~cm}$ to $11 \mathrm{~cm} .^{2,6,7}$

The false diverticula do not contain layers of muscularis or serosa and tend to form more often in the antrum. It is possible to classify as pulsation and traction diverticulum according to the formation mechanisms. Conditions such as obesity or chronic cough cause chronic increase in intragastric pressure, trigger pulsion diverticulum; where conditions associated with inflammatory processes such as surrounding peptic ulcer, malignant disease, pancreatitis, gastric outlet obstruction and patients who undergo to a bypass with Roux-Y gastric can develop traction gastric diverticulum. .,6,8 $^{5}$

Gastric diverticulum patients are generally asymptomatic or have ambiguous symptoms seen in other diseases of GIS, such as peptic ulcer or gastritis, so it is necessary to doubt this pathology for differential diagnosis. GD may not be visualized in upper GIS contrast radiographic studies and during endoscopy, especially when they are narrow-necked. In a large review, Palmer ${ }^{9}$ reported that $5 \%$ of GD are missed during upper gastrointestinal investigation.

The upper GIS endoscopy has the highest diagnostic value since it can directly see the high morbidity conditions such as ectopic mucosa, ulceration and malignancy in the diverticulum, and has the possibility of simultaneous biopsy. ${ }^{10,11}$ The upper GIS radiographs with barium, which have been used more frequently in the radiology routine in the past, can show gastric diverticulum with high accuracy when obtained with appropriate position and technique. It has been shown that during contrast radiographic series,

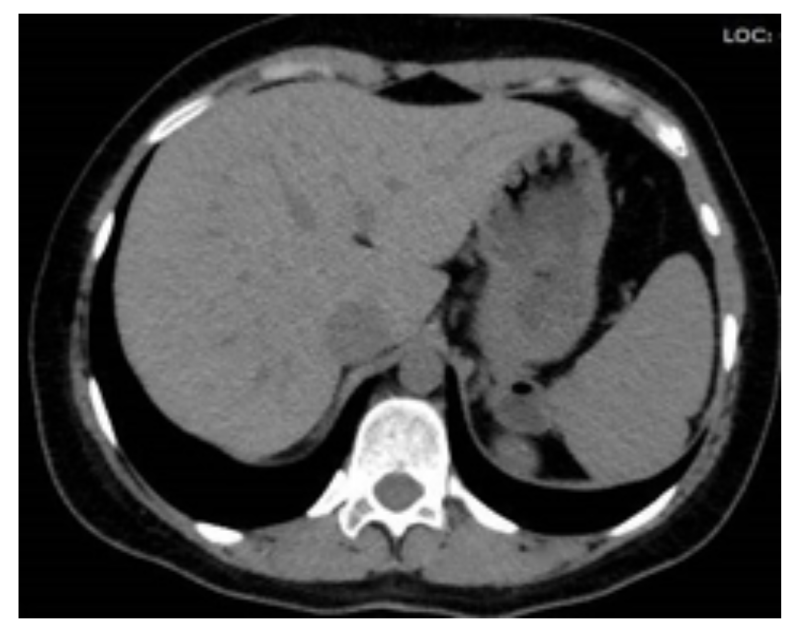

Figure 1b: CT-scan-axial sections, GD containing air 
the GD is best recognized with the use of a right, anterior oblique angle while the individual is in a trendelenburg and slightly left lateral decubitus position. . $^{12,13}$

Since CT scans are not able to show real time gastrointestinal tract movement, the peristaltic activity occurring in the stomach wall at the time of the examination causes false images and therefore diagnostic errors in daily practice. Sometimes radiologists misunderstand this normal gastric wall appearance. In order to diagnose gastric diverticulum with $\mathrm{CT}$, it is necessary to observe the continuity with gastric lumen and/or to observe the air density, if possible, to fill the diverticular lumen with given oral contrast agent. As most GD take part along the posterior wall of the stomach, it has been suggested that CT scans obtained in the prone position may facilitate air movement to the top and form an air-fluid level. ${ }^{14}$

However, CT scans are reported to mistake diverticula for adrenal masses ${ }^{15}$, we think, this usually depends on the experience of the radiologist. The fat line between the lateral adrenal crus can be seen with attention and also coronal or sagittal images are very helpful to distinguish between GD and left adrenal mass. The CT study is non-invasive and has much less radiation dose with advanced multislice 3D reformat technology. Major advantages of CT are the ability to show perigastric space, accompanying epigastric inflammation, abscess and perforation. Life threatening complications accompanying the diverticulum can be investigated and definitely reported with $\mathrm{CT}$ examination.

However, the methods used to detect GD occasionally fail, combined radiological and endoscopic diagnostic approach should be used. ${ }^{16}$

\section{Conclusion}

GD are one of the rarest anomalies of the GIS and are generally asymptomatic or cause vague symptoms, such as pain, discomfort, and dyspepsia. Although not very often, their diagnosis and followup are important because of potential risk for life threatening complications. First of all, suspicion of this pathology is required for the diagnosis, followed by combined use of radiological and endoscopic studies.

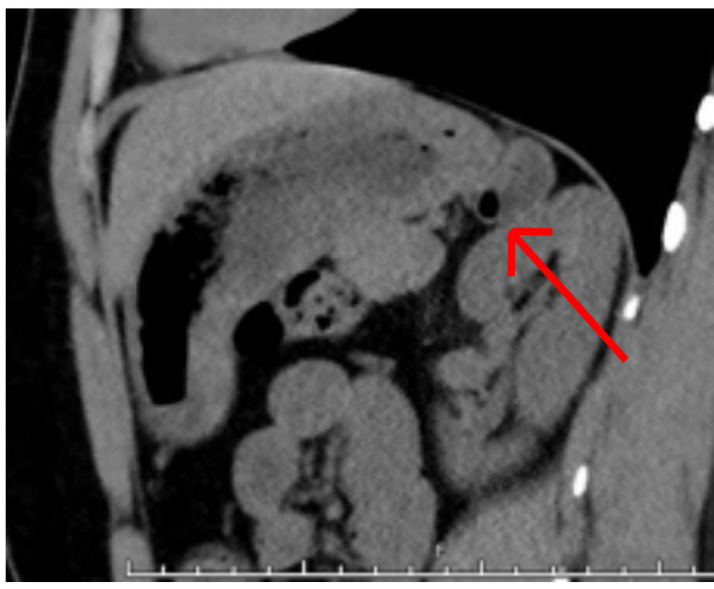

Figure 2: CT-scan-sagittal section observing diverticulum on the posterior wall of the stomach containing air (Arrow)

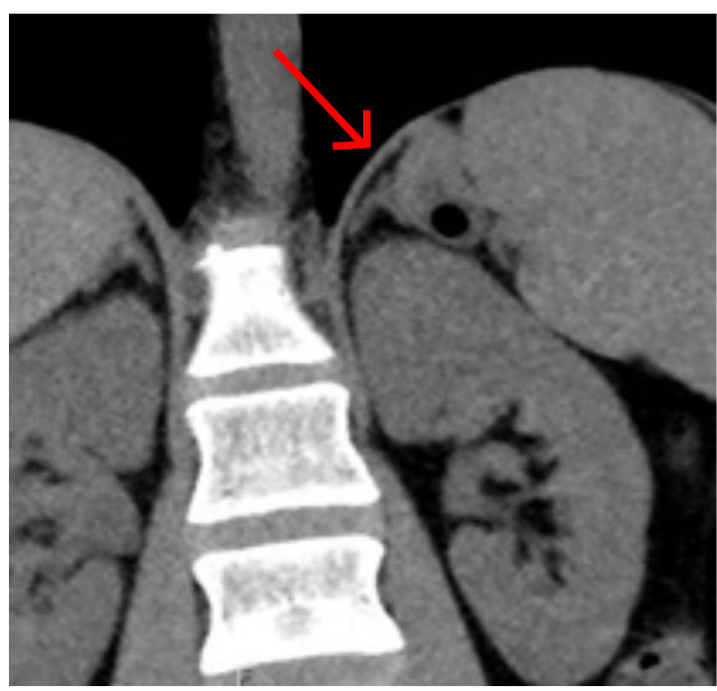

Figure 3: CT-scan-coronal section where saccular image is observed with air density inside. Thin fat line (Arrow) can be seen between the GD and left adrenal gland.

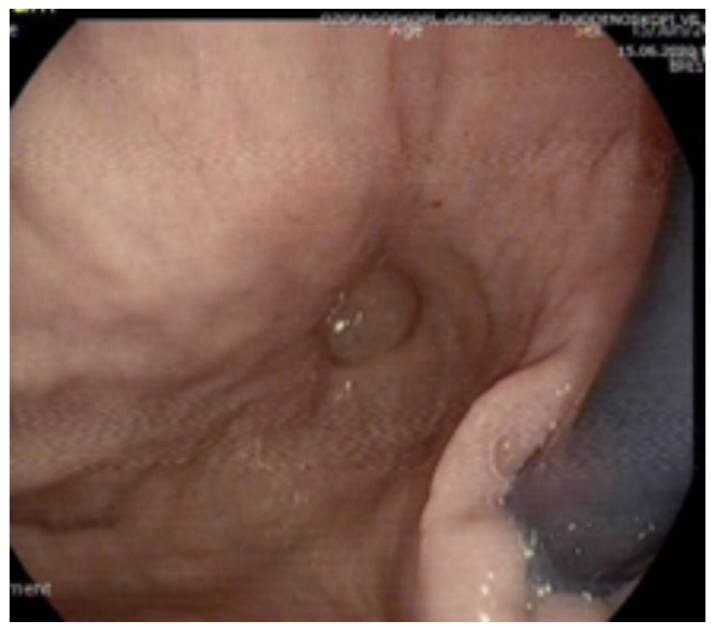

Figure 4: Endoscopic view of the gastric diverticulum located in the fundus of the stomach 


\section{Conflict of Interests}

The authors declared that there are no potential conflicts of interest with respect to the research, authorship, and/or publication of this article.

\section{References}

1. Gockel I, Thomschke D, Lorenz D. Gastric diverticula, J Gastroenterol Hepatol. 2004 Feb;19(2):227. doi: 10.1111/j.1440-1746.2004.3339b.x.

2. Donkervoort SC, Baak LC, Blaauwgeers JL, Gerhards MF. Laparoscopic resection of a symptomatic gastric diverticulum: a minimally invasive solution. JSLS. 2006 Oct-Dec;10(4):525-7.

3. Rodeberg DA, Zaheer S, Moir CR, Ishitani MB. Gastric diverticulum: a series of four pediatric patients. J Pediatr Gastroenterol Nutr. 2002 May;34(5):564-7. doi: 10.1097/00005176-200205000-00019.

4. DuBois B, Powell B, Voeller G. Gastric diverticulum: "a wayside house of ill fame" with a laparoscopic solution, JSLS. Jul-Sep 2012;16(3):473-7. doi: 10.4293/108680812X 13462882736330 .

5. Rashid F, Aber A, Iftikhar SY. A review on gastric diverticulum. World J Emerg Surg. 2012 Jan 18;7(1):1. doi: 10.1186/1749-7922-7-1.

6. Shah J, Patel K, Sunkara T, Papafragkakis C, Shahidullah A. Gastric Diverticulum: A Comprehensive Review. Inflamm Intest Dis. 2019 Apr;3(4):161-6. doi: 10.1159/000495463.

7. Podda M, Atzeni J, Messina Campanella A, Saba A, Pisanu A. Syncope with Surprise: An Unexpected
Finding of Huge Gastric Diverticulum. Case Rep Surg. 2016;2016:1941293. doi: 10.1155/2016/1941293.

8. Muis MO, Leitao K, Havnen J, Glomsaker TB, Søreide JA. Gastric diverticulum and halitosis-A case for surgery? Int J Surg Case Rep. 2014;5(7):431-3. doi: 10.1016/j. ijscr.2014.04.029.

9. Palmer ED. Gastric diverticula. Int Abstr Surg. 1951 May;92(5):417-28.

10. Wolters VM, Nikkels PG, Van Der Zee DC, Kramer PP, De Schryver JE, Reijnen IG, Houwen RH. A gastric diverticulum containing pancreatic tissue and presenting as congenital double pylorus: case report and review of the literature. J Pediatr Gastroenterol Nutr. 2001 Jul;33(1):8991. doi: 10.1097/00005176-200107000-00017.

11. Anaise D, Brand DL, Smith NL, Soroff HS. Pitfalls in the diagnosis and treatment of a symptomatic gastric diverticulum. Gastrointest Endosc. 1984 Feb;30(1):28-30. doi: 10.1016/s0016-5107(84)72291-7.

12. Bothén NF, Eklöf $O$. Diverticula and duplications (enterogenous cysts) of the stomach and duodenum. Am J Roentgenol Radium Ther Nucl Med. 1966 Feb;96(2):37581. doi: 10.2214/ajr.96.2.375.

13. Eras P, Beranbaum SL. Gastric diverticula: congenital and acquired. Am J Gastroenterol. 1972 Feb;57(2):120-32.

14. Feng YE, Zhang Z. Gastric diverticulum simulating a left adrenal mass: A case report and review of the literature. Oncol Lett. 2015 Oct;10(4):2477-80. doi: 10.3892/ ol.2015.3559.

15. Schwartz AN, Goiney RC, Graney DO. Gastric diverticulum simulating an adrenal mass: CT appearance and embryogenesis. AJR Am J Roentgenol. 1986 Mar;146(3):553-4. doi: 10.2214/ajr.146.3.553.

16. Simon M, Zuber-Jerger I, Schölmerich J. True gastric diverticulum. Dig Liver Dis. 2009 May;41(5):370. doi: 10.1016/j.dld.2008.06.016. 